\title{
OPTIMIZATION OF HERBICIDE TEANA 9EC DOSE FOR CONTROLLING WEEDS IN BRINJAL
}

\author{
S.K.Paul ${ }^{1}$, S.Mazumder ${ }^{2}$, T.A.Mujahidi ${ }^{3}$, S.K.Roy ${ }^{4}$ and S.Kundu ${ }^{5}$ \\ ${ }^{1}$ Agronomy Division, Bangladesh Agricultural Research Institute, Joydebpur, Gazipur-1701, Bangladesh \\ 2Sher-e-Bangla Nagar Adorsha Mohila College, Dhaka-1207, Bangladesh \\ ${ }^{3}$ Plant Breeding Division, Bangladesh Agricultural Research Institute, Joydebpur, Gazipur-1701, Bangladesh \\ ${ }^{4}$ Department of Genetics and Plant Breeding, Sher-e-Bangla Agricultural University, Dhaka-1207, Bangladesh \\ 5 Planning and Evaluation Division, Bangladesh Agricultural Research Institute, Joydebpur, Gazipur-1701, Bangladesh.
}

Key words: Herbicide, weed management, brinjal

\begin{abstract}
An experiment was conducted at the research field of Agronomy Division, Bangladesh Agricultural Research Institute, Joydebpur, Gazipur during the rabi season of 20132014 to find out the optimum dose of herbicide (Teana $9 \mathrm{EC}$ ) to control weeds in brinjal field for getting higher yield. Six treatments, viz.: (i) Teana 9 EC @ $750 \mathrm{ml} \mathrm{ha}^{-1}$ spraying on 15 DAS and 45 DAS, (ii) Teana 9EC @ $1000 \mathrm{ml} \mathrm{ha}^{-1}$ spraying on 15 DAS and 45 DAS, (iii) Teana 9EC @ $1250 \mathrm{ml} \mathrm{ha}^{-1}$ spraying on 15 DAS and 45 DAS, (iv) Teana 9EC @ $1500 \mathrm{ml} \mathrm{ha}^{-1}$ spraying on 15 DAS and 45 DAS, (v) two hand weddings, and (vi) no weeding (control) were used in the experiment. Among the weed species, Bathua (Chenopdium album), Durba (Cynodon dactylon), Anguli (Digitaria sanguinalis), Helencha (Jussiaea repens), Hatishur (Heliotropium indicum), Shama (Echinochola crusgalli), Swetlomy (Gnaphalium japonicum), Mutha (Cyperus rotundus), Shaknote (Amaranthus viridis), Gaicha (Paspalum commersonit), Chapra (Eleusine indica), Bon Masur (Vicia sativa) were found dominant in wheat field. The result showed that the highest dry weight of weeds at 30 and 60 DAS were 12.24 and $24.12 \mathrm{gm}^{-2}$ in control plot, whereas the lowest weed dry weight was observed in two hand weedings. The maximum weed control efficiency over control both at 30 and 60 DAS were 79.41 and $74.32 \%$, respectively in the treatment with two hand weedings. The highest number of fruits plant ${ }^{-1}$, single fruit weight $(\mathrm{g})$, fruit length $(\mathrm{cm})$ and yield $\left(\mathrm{t} \mathrm{ha} \mathrm{h}^{-1}\right)$ was found in the treatment having two hand weedings and the lowest in no weeding (control). Though the weed control efficiency (WCE) was the highest in the treatment with two hand weedings due to higher labor cost but the maximum benefit cost ratio was the highest when Teana 9EC @ $1500 \mathrm{ml} / \mathrm{ha}$ was sprayed two times on 15 and 45 DAS.
\end{abstract}

\section{Introduction}

In crop production there are many causes of yield loss, of which weed is one of the most important one which also deteriorates the quality of crops (Hasanuzzaman et al., 2007. Weeds cause enormous losses to crops even more than other pests worldwide. Oerke and Dehne (1997) found that weeds cause around 33\% of total crop loss in Asia and other countries. On an average, $37.3 \%$ of crop produce is damaged by weeds in Bangladesh (Karim et al., 1998) that valued approximately Tk. 59665.7 million (Karim, 2008). Production losses in Bangladesh due to weeds as 33.2\% in food crops, $41.3 \%$ in cereals, $31.9 \%$ in pulses, $40.8 \%$ in oilseed crops, $34.2 \%$ in fibre crops and $40.3 \%$ in rice (Paul et al., 2015). Hossain et al. (2012a) reported that the eighteen different weed species of which ten majors were observed much more serious to the crop. 
Paul et al.

Brinjal (Solanum melongena L.) is one of the most important, widely and round the year cultivated popular vegetable crop in Bangladesh. It grows well in winter season. But it can be grown in all seasons. Though it is grown well year round, but weed decreases the yield drastically. There are many factors responsible for low yield, of which weed reduces its yield if not properly controlled (Hasanuzzaman et al., 2008).Crop management practices play an important role in crop production of which weed control is an important task which involves a lot of production cost due to unavailability of human labor in the world as well as Bangladesh. As a result the alternate way to control weeds by the use of herbicide is gradually increased. The excess use of herbicide to control weeds is hazardous for health and causes environmental pollution. But lower doses cannot control the weeds properly. So, determination of optimum doses of herbicide is essential. The herbicides namely, Teana 9EC is a selective herbicide and effective for control of most broad leaf and sedge weeds in brinjal field. It is assumed that Teana 9EC may provide more effective, economic and easier solution for weed management in brinjal in comparison to manual weeding. Considering the above facts, the trial was undertaken to determine the optimum doses of herbicide (Teana 9EC) on weed control and subsequent growth and yield of brinjal.

\section{Materials and Methods}

The experiment was conducted at the Agronomy research field of Bangladesh Agricultural Research Institute, Gazipur during the period from November 2013 to May 2014. The land was medium high and the soil was clay loam in texture. Six treatments viz., (i) Teana 9EC @ $750 \mathrm{ml} \mathrm{ha}^{-1}$ spraying at 15 DAS (days after sowing) DAS and 45 DAS, (ii) Teana 9EC @ 1000 $\mathrm{ml} \mathrm{ha}^{-1}$ spraying at 15 DAS and 45 DAS, (iii) Teana 9EC @ $1250 \mathrm{ml} \mathrm{ha}^{-1}$ spraying at 15 DAS and 45 DAS, (iv) Teana 9EC @ $1500 \mathrm{ml} \mathrm{ha}^{-1}$ spraying at 15 DAS and 45 DAS, (v) Two hand weedings and (vi) No weeding were in the study. The unit plot size was $3 \mathrm{~m} \times 4 \mathrm{~m}$. The brinjal plant was transplanted on 26 November 2013. The brinjal field was fertilized with 80-60-40$20 \mathrm{~kg} \mathrm{~N}-\mathrm{P}_{2} \mathrm{O}_{5^{-}} \mathrm{K}_{2} \mathrm{O}-\mathrm{S}$ ha-1. Three irrigations were given at 21, 55 and 75 DAS. Brinjal (cv. BARI Begun 10) seedlings were transplanted maintaining $75 \mathrm{~cm} \times 60 \mathrm{~cm}$ spacing. Herbicides were sprayed with a Knapsack sprayer as per treatment and all other operations were done as and when required. Weed samples were collected at 30 and 60 DAS. Data on yield components were taken and analyzed statistically using MSTAT-C program.

The Relative Density (RD) and weed control Efficiency (WEC) were calculated by the following formula.

Relative Density $(\mathrm{RD})=\frac{\text { No of specific weed species }}{\text { Total no. of weeds }} \times 100$

Weed Control Efficiency (WEC) $=\frac{\text { Dry wt. of control plot }- \text { Dry wt. of specific plot }}{\text { Dry wt. of controlplot }} \times 100$

\section{Results and Discussion}

Major weeds flora were found in the experiment plots i.e. Bathua (Chenopdium album), Durba (Cynodon dactylon), Anguli (Digitaria sanguinalis), Helencha (Jussiaea repens), Hatishur (Heliotropium indicum), Shama (Echinochola crus-galli), Swetlomy (Gnaphalium japonicum), Mutha (Cyperus rotundus), Shaknote (Amaranthus viridis), Gaicha (Paspalum commersonii), Chapra (Eleusine indica), Bon Masur (Vcia sativa) (Table 1). No. of weeds and relative density of 
Optimization of Herbicide Teana 9EC Dose for Controlling Weeds in Brinjal

weeds were found maximum from sps. shama in all treatments except two hand weeding where maximum from weed species helencha (Table 1).

Table 1. Weed infestation in wheat field at 30 and 60 DAS during the 2013-2014

\begin{tabular}{|c|c|c|c|c|c|c|}
\hline \multirow[t]{2}{*}{ Treatment } & \multicolumn{2}{|r|}{ Weeds species } & \multicolumn{2}{|c|}{$\begin{array}{l}\text { No. of weeds } \\
/ \mathrm{m}^{2}\end{array}$} & \multicolumn{2}{|c|}{$\begin{array}{c}\text { Relative Density } \\
(\%)\end{array}$} \\
\hline & Local name & Scientific name & $\begin{array}{c}30 \\
\text { DAS }\end{array}$ & $\begin{array}{c}60 \\
\text { DAS }\end{array}$ & $\begin{array}{c}30 \\
\text { DAS }\end{array}$ & $\begin{array}{c}60 \\
\text { DAS }\end{array}$ \\
\hline $\mathrm{T} 1=\quad$ Teana & Bathua & Chenopdium album (L.) & 2 & 6 & 2.74 & 5.77 \\
\hline 9EC @ $750 \mathrm{ml}$ & Durba & Cynodon dactylon & 14 & 17 & 19.18 & 13.46 \\
\hline $\mathrm{ha}^{-1}$ spraying at & Anguli & Digitariasa nguinalis & 3 & 6 & 4.11 & 5.77 \\
\hline 15 and 45 & Helencha & Jussiaea repens & 3 & 7 & 4.11 & 6.73 \\
\hline \multirow[t]{10}{*}{ DAS } & Hatishur & Heliotropium indicum & 1 & 3 & 1.37 & 2.88 \\
\hline & Shama & Echinochola crus-galli & 25 & 32 & 34.24 & 30.77 \\
\hline & Bangchora & & 3 & 4 & 4.11 & 3.84 \\
\hline & Swelomy & Gnaphalium japonicum & 0 & 1 & 0 & 0.96 \\
\hline & Mutha & Cyperus rotundus & 3 & 7 & 4.11 & 6.73 \\
\hline & Shaknote & Amaranthus viridis & 1 & 2 & 1.37 & 1.92 \\
\hline & Gaicha & Paspalum commersonii & 2 & 1 & 2.74 & 0.96 \\
\hline & Chapra & Eleusine indica & 16 & 18 & 21.92 & 17.31 \\
\hline & Bon Masur & Vicia sativa & - & 2 & - & 1.92 \\
\hline & & Total & 73 & 104 & 100 & 100 \\
\hline $\mathrm{T} 2=$ Teana & Bathua & Chenopdium album (L.) & 1 & 2 & 2.13 & 2.78 \\
\hline 9EC @ 1000 & Durba & Cynodon dactylon & 5 & 9 & 10.64 & 12.50 \\
\hline $\mathrm{ml} \quad \mathrm{ha}^{-1}$ & Anguli & Digitaria sanguinalis & 0 & 3 & 0 & 4.17 \\
\hline spraying at 15 & Helencha & Jussiae arepens & 6 & 12 & 12.76 & 16.67 \\
\hline \multirow[t]{10}{*}{ and 45 DAS } & Hatishur & Heliotropium indicum & 0 & 3 & 0 & 4.17 \\
\hline & Shama & Echinochola crus-galli & 12 & 9 & 25.53 & 12.50 \\
\hline & Bangchora & & 0 & 2 & 0 & 2.98 \\
\hline & Swetlomy & Gnaphaliumj aponicum & 5 & 7 & 9.6 & 8.86 \\
\hline & Mutha & Cyperus rotundus & 11 & 14 & 18.41 & 19.64 \\
\hline & Shaknote & Amaranthus viridis & 3 & 7 & 5.38 & 9.92 \\
\hline & Gaicha & Paspalum comersonii & 1 & 3 & 2.13 & 4.37 \\
\hline & Chapra & Eleusine indica & 6 & 4 & 12.76 & 6.11 \\
\hline & Bon Masur & Vicia sativa & 2 & 4 & 4.26 & 5.55 \\
\hline & & Total & 52 & 79 & 100 & 100 \\
\hline $\mathrm{T} 3=$ Teana & Bathua & Chenopdium album (L.) & 6 & 8 & 13.95 & 15.68 \\
\hline 9EC @ 1250 & Durba & Cynodon dactylon & 5 & 6 & 11.65 & 11.76 \\
\hline mlha ${ }^{-1}$ spraying & Anguli & Digitaria sanguinalis & 2 & 3 & 4.65 & 5.89 \\
\hline at $15 \mathrm{DAS}$ and & Helencha & Jussiae arepens & 2 & 2 & 4.65 & 3.92 \\
\hline \multirow[t]{10}{*}{45 DAS } & Hatishur & Heliotropiu mindicum & 2 & 3 & 4.65 & 5.89 \\
\hline & Shama & Echinochola crus-galli & 10 & 8 & 23.25 & 15.68 \\
\hline & Bangchora & & 4 & 7 & 9.30 & 13.72 \\
\hline & Swetlomy & Gnaphalium japonicum & 1 & 2 & 2.27 & 3.84 \\
\hline & Mutha & Cyperus rotundus & 2 & 3 & 4.65 & 5.89 \\
\hline & Shaknote & Amaranthus viridis & 3 & 3 & 6.98 & 5.89 \\
\hline & Gaicha & Paspalum commersonii & - & 1 & - & 2.27 \\
\hline & Chapra & Eleusine indica & 8 & 8 & 18.60 & 15.68 \\
\hline & Bon Masur & Vicia sativa & 1 & 3 & 2.32 & 5.89 \\
\hline & & Total & 45 & 54 & 100 & 100 \\
\hline $\begin{array}{l}\text { T4 = Teana } \\
9 \mathrm{EC} @ 1500\end{array}$ & Bathua & Chenopdium album (L.) & 1 & 2 & 4.55 & 6.90 \\
\hline mlha-1 spraying & Durba & Cynodon dactylon & 1 & 3 & 4.55 & 10.34 \\
\hline
\end{tabular}


Paul et al.

\begin{tabular}{|c|c|c|c|c|c|c|}
\hline \multirow[t]{2}{*}{ Treatment } & \multicolumn{2}{|r|}{ Weeds species } & \multicolumn{2}{|c|}{$\begin{array}{l}\text { No. of weeds } \\
/ \mathrm{m}^{2}\end{array}$} & \multicolumn{2}{|c|}{$\begin{array}{c}\text { Relative Density } \\
\text { (\%) }\end{array}$} \\
\hline & Local name & Scientific name & $\begin{array}{c}30 \\
\text { DAS }\end{array}$ & $\begin{array}{c}60 \\
\text { DAS }\end{array}$ & $\begin{array}{c}30 \\
\text { DAS }\end{array}$ & $\begin{array}{c}60 \\
\text { DAS }\end{array}$ \\
\hline \multirow{12}{*}{$\begin{array}{l}\text { at } 15 \text { DAS and } \\
45 \text { DAS }\end{array}$} & Anguli & Digitaria sanguinalis & 1 & 3 & 4.55 & 10.34 \\
\hline & Helencha & Jussiaea repens & 5 & 3 & 22.72 & 10.34 \\
\hline & Hatishur & Heliotropium indicum & - & 2 & - & 6.90 \\
\hline & Shama & Echinochola crus-galli & 3 & 5 & 13.63 & 17.24 \\
\hline & Bangchora & & 1 & - & 4.55 & - \\
\hline & Swetlomy & Gnaphaliumj aponicum & - & 2 & - & 6.90 \\
\hline & Mutha & Cyperus rotundus & 6 & 5 & 27.27 & 17.24 \\
\hline & Shaknote & Amaranthus viridis & 4 & - & 18.18 & - \\
\hline & Gaicha & Paspalum commersonii & 2 & - & 6.90 & - \\
\hline & Chapra & Eleusine indica & - & 5 & - & 17.24 \\
\hline & Bon Masur & Vicia sativa & 1 & 2 & 4.55 & 6.90 \\
\hline & & Total & 25 & 30 & 100 & 100 \\
\hline Two & Bathua & Chenopdium album (L.) & 1 & 3 & 3.57 & 9.67 \\
\hline \multirow{13}{*}{ hand weeding } & Durba & Cynodon dactylon & 1 & 2 & 3.57 & 6.45 \\
\hline & Anguli & Digitaria sanguinalis & 1 & 1 & 3.57 & 3.22 \\
\hline & Helencha & Jussiaea repens & 11 & 8 & 39.29 & 25.82 \\
\hline & Hatishur & Heliotropium indicum & 1 & 2 & 3.57 & 6.45 \\
\hline & Shama & Echinochola crus-galli & - & 1 & - & 3.22 \\
\hline & Bangchora & & 4 & 3 & 14.29 & 9.68 \\
\hline & Swetlomy & Gnaphalium japonicum & 1 & 2 & 3.57 & 6.45 \\
\hline & Mutha & Cyperus rotundus & - & 3 & - & 9.68 \\
\hline & Shaknote & Amaranthus viridis & 4 & - & 14.29 & - \\
\hline & Gaicha & Paspalum commersonii & 1 & - & 3.57 & - \\
\hline & Chapra & Eleusine indica & 2 & 6 & 7.14 & 19.36 \\
\hline & Bon Masur & Vicia sativa & 1 & - & 3.57 & - \\
\hline & & Total & 28 & 31 & 100 & 100 \\
\hline $\mathrm{T} 6=$ & Bathua & Chenopdium album (L.) & 7 & 9 & 6.14 & 5.45 \\
\hline \multirow{13}{*}{ weeding } & Durba & Cynodon dactylon & 7 & 9 & 6.14 & 6.36 \\
\hline & Anguli & Digitaria sanguinalis & 10 & 15 & 8.77 & 14.55 \\
\hline & Helencha & Jussiaea repens & 6 & 8 & 5.26 & 7.28 \\
\hline & Hatishur & Heliotropium indicum & 4 & 8 & 3.51 & 6.36 \\
\hline & Shama & Echinochola crus-galli & 31 & 26 & 27.19 & 22.73 \\
\hline & Bangchora & & 14 & 2 & 12.29 & 1.81 \\
\hline & Swetlomy & Gnaphalium japonicum & 1 & 2 & 0.88 & 1.81 \\
\hline & Mutha & Cyperus rotundus & 8 & 12 & 7.01 & 10.91 \\
\hline & Shaknote & Amaranthus viridis & 6 & 8 & 5.26 & 7.28 \\
\hline & Gaicha & Paspalum commersonii & 2 & 2 & 1.75 & 0.91 \\
\hline & Chapra & Eleusine indica & 12 & 10 & 10.54 & 10 \\
\hline & Bon Masur & Vicia sativa & 6 & 8 & 5.26 & 6.36 \\
\hline & & Total & 114 & 119 & 100 & 100 \\
\hline
\end{tabular}

Dry weights of weeds were significantly influenced by different weeding methods (Table 2). The highest dry weight of weeds at 30 and 60 DAS were 12.24 and $24.12 \mathrm{gm}^{-2}$ in control plot where lowest in two hand weedings. The maximum weed control efficiency over control both at 30 and 60 DAS were 79.41 and $74.32 \%$, respectively in treatment two hand weedings. Though the weed control efficiency (WCE) was highest in two hand weedings but due to higher cost of labor, the maximum benefit cost ratio was highest in Teana 9EC @ $1500 \mathrm{ml} \mathrm{ha}^{-1}$ spraying at 15 and 45 DAS. Results were in agreement with Hossain et al. (2012a), Hossain et al. (2012b) and Islam (2014). 
Optimization of Herbicide Teana 9EC Dose for Controlling Weeds in Brinjal

Table 2. Dry weight and weed control Efficiency in brinjal field at 30 and 60 DAS as affected by different doses of Teana $9 \mathrm{EC}$

\begin{tabular}{|c|c|c|c|c|}
\hline \multirow[t]{2}{*}{ Treatments } & \multicolumn{2}{|c|}{ At 30 DAS } & \multicolumn{2}{|c|}{ At 60 DAS } \\
\hline & $\begin{array}{l}\text { Dry weight of } \\
\text { weeds } \mathrm{m}^{-2}(\mathrm{~g})\end{array}$ & $\begin{array}{l}\text { Weed control } \\
\text { Efficiency (\%) }\end{array}$ & $\begin{array}{l}\text { Dry weight of } \\
\text { weeds } \mathrm{m}^{-2}(\mathrm{~g})\end{array}$ & $\begin{array}{l}\text { Weed control } \\
\text { Efficiency (\%) }\end{array}$ \\
\hline $\begin{array}{l}\text { T1 = Teana 9EC @ } 750 \\
\text { ml ha-1 spraying at } 15 \text { and } \\
45 \text { DAS }^{-1}\end{array}$ & 8.72 & 28.76 & 18.39 & 16.86 \\
\hline $\begin{array}{l}\text { T2= Teana 9EC @ } 1000 \\
\text { ml ha }{ }^{-1} \text { spraying at } 15 \text { and } \\
45 \text { DAS }\end{array}$ & 5.64 & 53.92 & 12.24 & 44.66 \\
\hline $\begin{array}{l}\text { T3 = Teana 9EC @ } 1250 \\
\text { ml ha }{ }^{-1} \text { spraying at } 15 \text { and } \\
45 \text { DAS }\end{array}$ & 3.12 & 74.51 & 7.76 & 64.91 \\
\hline $\begin{array}{l}\text { T4 = Teana 9EC @ } 1500 \\
\mathrm{ml} \mathrm{ha}^{-1} \text { spraying at } 15 \text { and } \\
45 \text { DAS }\end{array}$ & 2.88 & 76.47 & 6.57 & 70.29 \\
\hline $\mathrm{T} 5=$ Two hand weedings & 2.52 & 79.41 & 5.68 & 74.32 \\
\hline T6 $=$ No weeding & 12.24 & - & 22.12 & - \\
\hline
\end{tabular}

The maximum number of fruits plant ${ }^{-1}$, single fruit weight $(\mathrm{g})$, fruit length $(\mathrm{cm})$, yield $\left(\mathrm{t} \mathrm{ha}^{-1}\right)$ was found in two hand weedings followed by T4 and T3 treatment and lowest in no weeding. There was trend to increase yield with the increase of herbicides dose upto $1250 \mathrm{ml} \mathrm{ha}^{-1}$ (Table 3). Hossain (2013) reported that due to improper weed management crop losses occur from 30$80 \%$. In cereals and vegetables, hand weeding and use of mechanical weeder are often used, but now a day's use of herbicides are expanding quickly due to labour shortage and commercialization. Two hand weeding showed higher gross return but higher cost was involved in the treatment (Table 4). As a result higher gross return was incurred from treatment T4 with higher benefit cost ratio (1.26). So, sustainable weed management is very much effective for friendly-environment and biodiversity (control).

Table 3. Fruit yield and yield contributing characters of brinjal as affected by different weed management practices

\begin{tabular}{l|c|c|c|c}
\hline Treatment & $\begin{array}{c}\text { Fruit plant } \\
\text { (no.) }\end{array}$ & $\begin{array}{c}\text { Single fruit } \\
\text { weight }(\mathrm{g})\end{array}$ & $\begin{array}{c}\text { Fruit length } \\
(\mathrm{cm})\end{array}$ & $\begin{array}{c}\text { Fruit yield } \\
\left(\mathrm{t} \mathrm{ha}^{-1}\right)\end{array}$ \\
\hline $\begin{array}{l}\text { T1 = Teana 9EC @ 750 } \mathrm{ml} \mathrm{ha}^{-1} \\
\text { spraying at 15 and 45 DAS }\end{array}$ & 11.05 & 86.48 & 24.09 & 14.56 \\
T2 = Teana 9EC @ 1000 ml ha- & 14.99 & 86.49 & 24.81 & 24.25 \\
$\begin{array}{l}\text { 1 spraying at 15 and 45 DAS } \\
\text { T3 = Teana 9EC @ 1250 ml ha-1 }\end{array}$ & 16.85 & 86.88 & 24.92 & 27.52 \\
spraying at 15 and 45 DAS & & & & \\
T4 = Teana 9EC @ 1500 ml ha- & 16.87 & 86.89 & 24.93 & 27.51 \\
1 & & & & \\
T5raying at 15 and 45 DAS & 17.36 & 87.53 & 24.91 & 28.47 \\
T6 No hand weedings & 5.85 & 86.11 & 24.65 & 8.31 \\
\hline LSD (0.05) & 0.59 & 2.66 & 0.16 & 1.28 \\
CV (\%) & 1.79 & 1.61 & 0.33 & 2.37 \\
\hline
\end{tabular}


Paul et al.

Table 4. Cost and Benefit analysis of brinjal as influenced by different weed control management practice

\begin{tabular}{|c|c|c|c|c|}
\hline Treatments & $\begin{array}{l}\text { Gross return } \\
(\text { Tk. ha-1) }\end{array}$ & $\begin{array}{l}\text { Total cost } \\
(\text { Tk. ha-1) }\end{array}$ & $\begin{array}{l}\text { Gross margin } \\
(\text { Tk. ha-1) }\end{array}$ & $\mathrm{BCR}$ \\
\hline $\begin{array}{l}\text { T1 = Teana 9EC @ } 750 \mathrm{ml} \mathrm{ha}^{-} \\
1_{\text {spraying at } 15 \text { and } 45 \text { DAS }}\end{array}$ & 107,000 & 92,300 & 14,700 & 1.16 \\
\hline $\begin{array}{l}\mathrm{T} 2=\text { Teana } 9 \mathrm{EC} @ 1000 \mathrm{ml} \mathrm{ha}^{-} \\
1_{\text {spraying at } 15} \text { and } 45 \text { DAS }\end{array}$ & 111,000 & 92,500 & 18,500 & 1.20 \\
\hline $\begin{array}{l}\text { T3 = Teana 9EC @ } 1250 \mathrm{ml} \mathrm{ha}^{-} \\
1_{\text {spraying at } 15 \text { and } 45 \mathrm{DAS}}\end{array}$ & 114,400 & 92,700 & 21,700 & 1.23 \\
\hline $\begin{array}{l}\mathrm{T} 4=\text { Teana } 9 \mathrm{EC} @ 1500 \mathrm{ml} \\
\text { ha }^{-1} \text { spraying at } 15 \text { and } 45 \mathrm{DAS}\end{array}$ & 116,600 & 92,900 & 23,700 & 1.26 \\
\hline $\mathrm{T} 5=$ Two hand weedings & 118,300 & 95,000 & 23,300 & 1.24 \\
\hline $\mathrm{T} 6=$ No weeding & 95,000 & 89,900 & 5,100 & 1.06 \\
\hline
\end{tabular}

\section{Conclusion}

From the above findings it may be concluded that Teana 9EC herbicide could be effectively control weeds of brinjal. Though the weed control efficiency (WCE) was the maximum in the treatment with two hand weedings but involved higher labor cost As such, the maximum benefit cost ratio was obtained from herbicides Teana 9EC @ $1500 \mathrm{ml} /$ ha sprayed two times as on 15 and 45 DAS.

\section{References}

Hasanuzzaman, M., K. Nahar and M.R. Karim. 2007. Effectiveness of different weed control methods on the performance of transplanted rice. Pak. J. Weed Sci. Res. 13: 17-25.

Hossain, M. S., M. M.Islam, A.T.M. M.Alam, I.Ahmed and M.J. Alam. 2012b. Species identification, density evaluation and green weight of weeds in Deshi jute (CorchoruscapsularisL.) growing areas of Bangladesh. Bangladesh J. Weed Sci. 3 (1\&2): 47-52.

Hossain, M. S., M. M.Islam, I. Ahmed, A.T.M.M.Alamand M.S. H. Bhuiyan. 2012a. Identification of species, density evaluation and green weight of weeds in Tossa jute (CorchorusolitoriusL.) growing areas of Bangladesh. Bangladesh J. Weed Sci. 3 (1\&2): 1924.

Hossain, S.T. 2013. Weed In Bangladesh: Issues and Challenges. (Key note paper), SOUVENIR, $4^{\text {th }}$ Conference of Weed Science Society of Bangladesh, Held on 03 May 2013 at Bangladesh Agril. Res. Council. pp. 22-28.

http://www.ejournalofscience.org/Download April14 pdf 4.php

Islam, M. M. 2014. Research Advances of Jute Field Weeds in Bangladesh: A Review. ARPN J. Sci. Technol. 4(4): 254-268.

Karim, S. M. R. 2008. Weeds and their impacts on biosecurity, environment and food security. Key note paper of $1^{\text {st }}$ National Conference and Seminar on Weeds and Food Security, 8 November: $5-18$. 
Optimization of Herbicide Teana 9EC Dose for Controlling Weeds in Brinjal

Karim, S.M.R., T.M.T. Iqbal and N. Islam.1998. Relative yields of crops and crop losses due to weed competition in Bangladesh. Pakistan J. Sci. Ind. Res. 41(6): 318-324.

Oerke, E.C. and H.W. Dehne.1997. Global crop production and the efficiency of crop protection: current situations and future trends. European J. Plant Pathol. 103(3): 203-215.

Paul, S.K., T.A.Mujahidi., M. H. Khan., S.K. Roy. and M.M. Rahman. 2015. Determination of the optimum doses of herbicide (Teana 9EC) to control weeds in wheat field. Bangladesh J. Weed Sci. 4 \& 5:93-99. 